\title{
PHOTOELECTRON SPECTROSCOPY BY SYNCHROTRON RADIATION
}

\author{
P.O. NILSSON \\ Department of Physics, Chalmers University of Technology, 41296 Göteborg, Sweden
}

The present understanding of the photoemission effect is reviewed. Simple and more advanced models are used for interpreting photoelectron spectroscopy data from valence bands and core levels in solids. A few experimental results are presented to illustrate the present status of the technique.

PACS numbers: $73.20 . \mathrm{Dx}, 79.60 .-\mathrm{i}$

\section{Introduction}

The properties of a solid are determined by its geometric, electronic and vibrational structure. In particular the electronic structure plays a key role for the understanding of the behaviour of solids. The most powerful method for studying the electronic structure is photoelectron spectroscopy (PES). With this technique the sample is irradiated with monochromatic photons and the emitted electrons analysed with respect to their kinetic energies. In the present paper we analyse models used to extract detailed information about electronic states from photoemission data. In particular crystals and their surfaces will be discussed. A few experimental results will be presented, mainly from our research group. We leave most of the applications and more detailed referencing to other authors at this school.

In Fig. 1 the relevant energies in the photoemission process are defined. The Fermi level $E_{\mathrm{F}}$ and the vacuum level $E_{\mathrm{V}}$ of the sample are indicated. Using monochromatic radiation of energy $h \nu$, electrons may be excited above the vacuum level of the solid. There is then a certain probability for an electron to escape out of the sample. Using energy conservation the measured kinetic energy of the electron in vacuum $E_{\text {kin }}$ can be converted to the binding energy $E_{\mathrm{B}}$. In a metal $E_{\mathrm{B}}$ is referred to the Fermi level and is given by

$$
E_{\mathrm{B}}=h \nu-e \phi-E_{\mathrm{kin}},
$$

where $e \phi=E_{\mathrm{V}}-E_{\mathrm{F}}$ is the work function of the sample. For a non-metal $E_{\mathrm{B}}$ is usually referred to the top of the valence band. Although Fig. 1 refers to a one-electron description, relation (1) is general. The interpretation of $E_{\mathbf{B}}$ is more complex than Fig. 1 indicates. This will be further discussed in Sect. 5. 


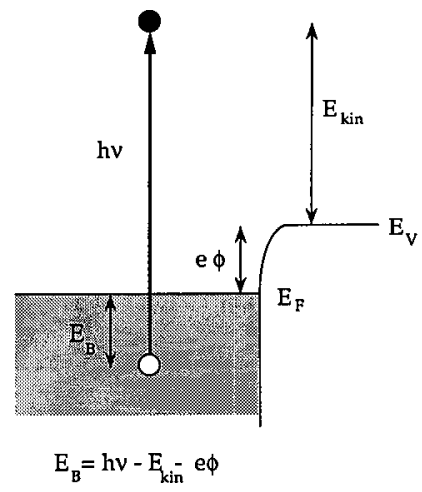

Fig. 1. Energy diagram for the photoelectron process. The electron binding energy can be derived from the knowledge of the photon energy $h \nu$, the kinetic energy of the electron in vacuum $E_{\text {kin }}$, and the work function $e \phi$ of the sample, see Eq. (1).

The photoelectric effect was discovered more than a hundred years ago, and its principle was explained by Einstein already in 1905 [1]. It is however only during the last 25 years that it has been utilised for detailed studies of the electronic structure of solids. The possibility to vary the photon energy continuously using synchrotron radiation has been of particular importance. Technical development is still going on and photoelectron spectroscopy is expected to be applied in new areas in the near future.

\section{The electronic structure of solids}

\subsection{The ground state}

The foundations for the theory of the electronic structure of matter were laid down in the papers by Hohenberg, Kohn, and Sham [2-4] in the middle of the 1960 's. These authors showed that the electronic ground state can be described exactly by one-body equations using an effective local potential

$$
\left\{-\frac{\hbar^{2} \nabla^{2}}{2 m}+V_{\mathrm{C}}(r)+V_{\mathrm{XC}}\right\} \Psi_{i}(r)=\varepsilon_{i} \psi(r) .
$$

Here $V_{\mathrm{C}}(r)$ is the sum of the Coulomb potential from all the nuclei and the average potential from all the electrons. $V_{\mathrm{XC}}(r)$ represents the exchange-correlation potential, which describes the effect of the correlated movements among the electrons, i.e. the deviation from the average effect. The formalism is referred to as the density functional theory (DFT). It states in particular that the total ground state energy of the sample can be expressed as a universal functional of the one-electron density $\rho(\boldsymbol{r})$ :

$$
E_{\mathrm{tot}}[\rho]=T[\rho]+\int \mathrm{d}^{3} r v_{\text {ion }}(r) \rho(r)+\int \mathrm{d}^{3} r \mathrm{~d}^{3} r^{\prime} \rho(r) \rho\left(r^{\prime}\right) /\left|r-r^{\prime}\right|+E_{\mathrm{XC}}[\rho] .
$$


Here $T[\rho]$ is the kinetic energy of the non-interacting electrons and $v_{\text {ion }}(r)$ the Coulomb potential from the nuclei. The exchange-correlation energy $E_{\mathrm{XC}}$ is related to $V_{\mathrm{XC}}(r)$ through $V_{\mathrm{XC}}(r)=\delta E_{\mathrm{XC}}[\rho] / \delta \rho(r)$.

A common approximation to DFT is the local density approximation (LDA). Here one assumes that the solid can be approximated by small boxes, each containing a homogeneous, interacting electron gas of appropriate average density. The LDA has been successfully applied to a variety of systems, even to situations where the density variations are not small, e.g. at surfaces. The DFT and the LDA can strictly only be used to calculate the electron density, total ground state energy and properties derived from these entities. The individual eigenvalues $\varepsilon_{i}$ in Eq. (2) have no direct physical interpretation but are quantities obtained at the density calculations. In many cases however $\varepsilon_{i}$ are good approximations to excitation energies as measured in e.g. photoemission, a fact which will be further discussed below.

\subsection{The excited state}

\subsubsection{Valence states}

The eigenvalues of Eq. (2) for the valence electrons in a crystal constitute the conventional band structure $\varepsilon_{n}(k)$, which usually is calculated in the LDA. An electron state (Bloch state) is characterised by the crystal momentum $\hbar k$ and the band index $n$. Although the $\varepsilon_{n}(k)$ data often has been successfully interpreted as excitation energies, as observed in e.g. PES, we should keep in mind that this approximation may fail. Rigorously, excited states are derivable from

$$
\left[-\frac{\hbar^{2} \nabla^{2}}{2 m}+V_{\mathrm{C}}(\boldsymbol{r})\right]+\phi_{i}(\boldsymbol{r})+\int \mathrm{d}^{3} \boldsymbol{r}^{\prime} \Sigma\left(\boldsymbol{r}, \boldsymbol{r}^{\prime} ; E_{i}\right) \phi_{i}\left(\boldsymbol{r}^{\prime}\right)=E_{i} \phi_{i}(\boldsymbol{r}) \text {. }
$$

Contrary to Eq. (2), Eq. (4) has no exact solutions. The self-energy $\Sigma\left(r, r^{\prime} ; E_{i}\right)$ is a non-local, energy dependent, and non-Hermitian operator. The energy dependence of the real and imaginary parts is shown in Fig. 2 for a free electron gas with the density of aluminium $\left(r_{\mathrm{s}}=2.07\right)$ [5]. Because of the translational invariance for free electrons there is in this case a local dependence on the momentum. At the Fermi level the real part $\Sigma_{\mathrm{r}}$ is equal to $V_{\mathrm{XC}}$ in Eq. (2) and amounts to $-\mathbf{5}$ to $-10 \mathrm{eV}$ for most materials. As seen, $\Sigma_{\mathrm{r}}$ is fairly constant below the Fermi level and in fact quite close to the constant value used in LDA band calculations. At high energies $\Sigma_{\mathrm{r}}$ approaches zero because response functions in general tend to zero for infinite energies and momenta.

The fact that $\Sigma$ is non-Hermitian gives rise to an imaginary part $\Sigma_{\mathrm{i}}$, which is related via dispersion relations to $\Sigma_{\mathrm{r}}$. If $\Sigma_{\mathrm{i}}$ is not too large, it is meaningful to define a quasi-particle (electron or hole with its screening cloud) described by the spectral function

$$
A(k, E)=\frac{1}{\pi} \frac{\left|\Sigma_{\mathrm{i}}(k, E)\right|}{\left[E-E_{k}-\Sigma_{\mathrm{r}}(k, E)\right]^{2}+\left[\Sigma_{\mathrm{i}}(k, E)\right]^{2}} .
$$

(For simplicity we have considered a local self-energy.) The Lorentzian peaks at $E=E_{k}-\Sigma_{\mathrm{r}}(k, E)$, meaning that the quasi-particle energy $E$ is shifted away from 


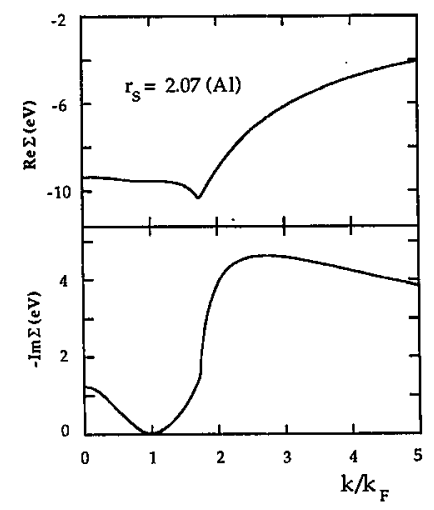

Fig. 2. The real and imaginary part of the self-energy for a free electron gas with a density corresponding to that of aluminum $\left(r_{s}=2.07\right)$. The data has been taken from Ref. [5].

the one-electron energy $E_{k}$ by the real part of the self-energy $\Sigma_{\mathrm{r}}$. The inverse width $\left(2 \Sigma_{\mathrm{i}}\right)^{-1}$ of the peak represents the lifetime of the quasi-particle. It results from inelastic scattering of the hole or electron with electrons. Figure 2 shows that $2 \Sigma_{\mathrm{i}}$ amounts to about $10 \mathrm{eV}$ for photoelectrons at high energies (above the plasmon energy). The associated mean free path for inelastic scattering is of the order of 5-10 $\AA$ in that region. As seen, valence holes typically have a width of a few tenths of an eV only.

We may incorporate the self-energy effects in a band structure. The real part $\Sigma_{\mathbf{r}}$ shifts the electron bands and $\Sigma_{\mathrm{i}}$ causes a broadening. This way we may keep an effective one-electron picture of photoemission. An effective one-body approximation does not always hold however for valence bands. For instance, strong correlation effects may introduce extra structure in the spectral function $A(k, E)$, such as satellites. We also mention that the non-locality of $\Sigma$ can have important contributions in e.g. semiconductors. For instance, the band gap and the band off-set at interfaces are correctly described only when this effect is included.

\subsubsection{Core states}

As mentioned, the excitation of extended valence states can usually be described in an effective one-electron picture. The electron states are then occupation-independent and the rest of the system is passive ("frozen"). For core electron excitations this picture breaks down. To describe photoemission from core states we have to abandon the one-electron picture and consider the conservation of the energy of the total system

$$
E_{\mathrm{f}}^{\mathrm{tot}}+E_{\mathrm{kin}}-E_{\mathrm{i}}^{\mathrm{tot}}=h \nu
$$

$E_{\mathrm{i}}^{\text {tot }}$ is the total ground state energy of the solid. We found above that the excitation shift of a valence state is of the order of $0.1 \mathrm{eV}$. The effect for a core hole is dramatically larger. Due to relaxation effects around the localised core hole the measured binding energy $E_{\mathrm{B}}$ may be several $\mathrm{eV}$ smaller than predicted by Eq. (2). 
We write

$$
E_{\mathrm{B}}=h \nu-E_{\mathrm{kin}}-e \phi=E_{\mathrm{f}}^{\mathrm{tot}}-E_{\mathrm{i}}^{\mathrm{tot}}-e \phi=\varepsilon_{\mathrm{i}}-E_{\text {relax }},
$$

where $\varepsilon_{\mathrm{i}}$ are one-electron energies derived from Eq. 2 and referred to the Fermi level. We note that the measured binding energy $E_{B}$ is dependent on both the initial and final state total energies. Note that the concept of "final state" was differently used above for the one-electron description of valence bands. There it referred to an electron state as described by the unoccupied band structure. For core hole excitation the final state refers to the total final state, where the core hole plays an important role.

\section{Phenomenology and experiment}

Recording the number $N(E)$ of photoemitted electrons per energy interval as a function of their kinetic energy $E_{\text {kin }}$ results in a so-called energy distribution curve (EDC). The measured $E_{\text {kin }}$ can be converted into the binding energy $E_{\mathrm{B}}$ using Eq. (1). There are alternative schemes to record the photoemitted electrons from valence states. In one method the electron detection energy is changed at the same rate as the photon energy is varied. This is called the constant initial spectroscopy (CIS). If the electron detection energy is kept constant when the photon energy is varied we have the constant final state spectroscopy (CFS). The most common mode, however, is to record EDC's. Also, technically this is convenient because the monochromator setting is fixed.

In addition to the energy of the external-photoelectron, the momentum may be measured. This is realised in a so-called angle-resolved experiment. Figure 3

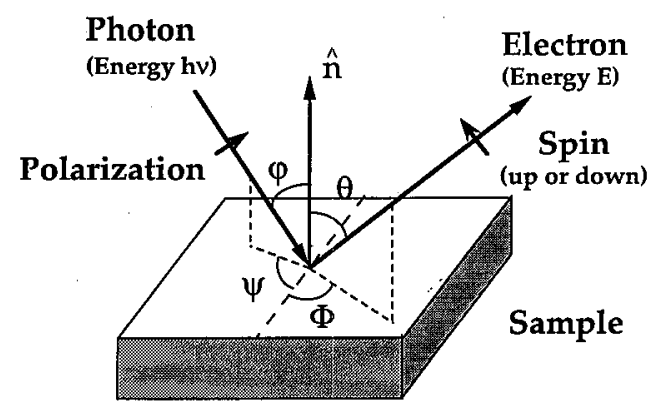

Fig. 3. The geometry of an angle-resolved photoemission experiment. There are in total eight parameters to specify. Often however only the photon energy $h \nu$, the electron energy $E_{\text {kin }}$, and the detection angles $(\theta, \varphi)$ arc given.

shows how the angles of the incident photon and the detected electron may be specified. Sometimes also the polarization of the photon and the spin of the electron is determined. In total we have eight parameters to specify in an angle-resolved experiment. 


\section{Photoelectron spectroscopy on valence states}

\subsection{Simple models}

It is usually enough to analyse photoemission data using fairly simple models. We will first discuss the kinetics in the case of excitation of valence electrons in a single crystal. We assume that the electron states can be described in a band structure formalism. The so-called "three-step model" is illustrated in Fig. 4. In the

\section{3 step model}
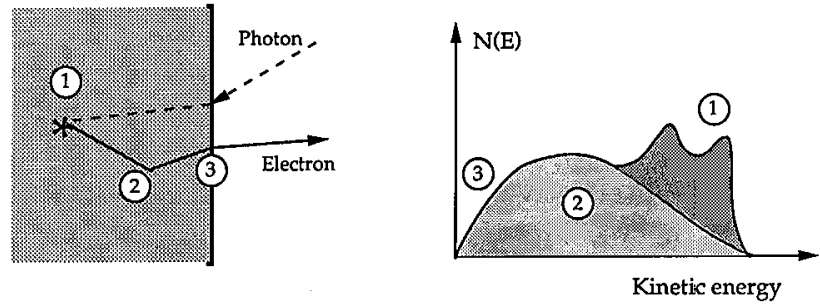

Fig. 4. The three-step model of photoemission: excitation (1), transport (2), and escape through the surface (3).

first step a photon of energy $h \nu$ penetrates into the crystal and excites an electron from a Bloch state $\psi_{\mathrm{i}}\left(\boldsymbol{k}_{\mathrm{i}}\right)$ into a Bloch state $\psi_{\mathrm{f}}\left(\boldsymbol{k}_{\mathrm{f}}\right)$. The momentum of the photon is small in the VUV range, therefore the electron crystal momentum $\hbar k$ is conserved within a reciprocal crystal momentum $\hbar g$. It may also happen that a surface state is excited, in which case the crystal momentum conservation is restricted to the momentum parallel to the surface, $k_{\|}$. In the second step the excited electron travels through the solid under inelastic electron-electron interactions. The energy losses result in a smooth background of "secondary" electrons. Finally, in the third step the electron escapes through the surface barrier into the vacuum under conservation of crystal momentum parallel to the surface.

We are here interested in the elastic ("primary") part of the EDC which is connected with the excitation process. Energy and momentum conservation results in

$$
\begin{aligned}
& E_{\mathrm{f}}\left(k_{\mathrm{f}}\right)-E_{\mathrm{i}}\left(k_{\mathrm{i}}\right)=h \nu \\
& k_{\mathrm{f}}=k_{\mathrm{i}}+g
\end{aligned}
$$

where $g$ is a reciprocal lattice vector. In a reduced zone scheme we have so-called vertical transitions, $k_{\mathrm{f}}=k_{\mathrm{i}}$. The momentum of the photon can be neglected in the VUV region. According to the "golden rule" the internal primary electron energy distribution can be written as

$$
N(E, h \nu)=C_{1} \int|M|^{2} \mathrm{~d}^{3} k \delta\left(E_{\mathrm{f}}(k)-E_{\mathrm{i}}(k)-h \nu\right) \delta\left(E_{\mathrm{f}}(k)-E\right) .
$$


Here $|M|^{2}$ is the transition probability given by the matrix element

$$
M=C_{2} \int \mathrm{d}^{3} r \psi_{\mathrm{f}} *(\boldsymbol{k}, \boldsymbol{r}) \Delta \psi_{\mathrm{i}}(\boldsymbol{k}, \boldsymbol{r}),
$$

where $\Delta=e p$ is the dipole operator, $e$ is the electric vector, and $p=-\mathrm{i} \hbar \nabla$ is the momentum operator.

We illustrate with a nearly free-electron (NFE) band structure, where the bands in the middle of the zone are well described by free-electron parabolas

$$
\begin{aligned}
& E_{\mathrm{f}}(k)=\hbar^{2}|k-g|^{2} / 2 m, \\
& E_{\mathrm{i}}(k)=\hbar^{2}|k|^{2} / 2 m .
\end{aligned}
$$

The direct transitions take place on an "optical surface" in $k$-space defined by one of the $\delta$-function in Eq. $(9)$, i.e. $E_{\mathrm{f}}(k)-E_{\mathrm{i}}(k)=h \nu$. Using Eqs. (11a) and (11b) the optical surface is described by

$$
k g=|g|^{2} / 2-m h \nu / \hbar^{2} .
$$

The constant projection of $k$ onto the $g$-vector generates a plane perpendicular to the $g$-vector, see Fig. $5 \mathrm{~b}$. In our approximation the matrix element $M$.
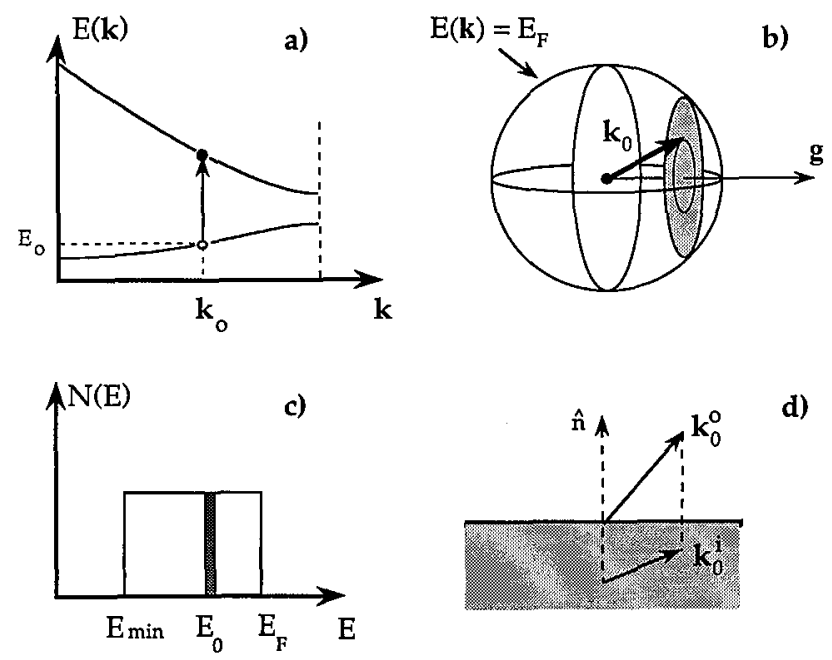

d)

Fig. 5. The angle-resolved photoemission experiment on a nearly free electron crystal; (a) the band structure, (b) optical energy surface, (c) energy distribution curve (EDC), and (d) escape process through the surface.

is constant, which results in a constant internal distribution $N(E)$, see Fig. 5c. Electrons with a specific energy $E_{0}$ are found on the electron $k$-surface defined by $E_{\mathrm{f}}(k)=E_{0}$, which is a sphere. It intersects the optical surface along the "photoemission line", which in our model is a circle. Equation (10) can be rewritten as an integration along this line

$$
N(E)=C_{3} \int \frac{\mathrm{d} l}{\left|\nabla E_{\mathrm{i}}(k) \times \nabla E_{\mathrm{f}}(k)\right|} .
$$


The EDC will consist of a number of broadened $\delta$-functions corresponding to direct transitions.

At the escape of the excited electron through the surface barrier the parallel crystal momentum of the electron is conserved, $\boldsymbol{k}_{\|}^{\mathrm{i}}=k_{\|}^{0}$, see Fig. $5 \mathrm{~d}$. In an angle-resolved experiment we measure the kinetic energy of the photoelectrons in vacuum $E_{\text {kin }}$ and the emission angle relative to the surface normal $\theta$. We have the relationship

$$
k_{\|}=\sqrt{2 m E_{\text {kin }}} \sin \theta / \hbar \text {. }
$$

It should be clear from our discussion that in an angular-resolved experiment we are able to verify a calculated band structure $E(k)$. In fact the first angular-resolved photoemission experiment was performed on the free-electron-like bands of silver and analysed according to the described model [6]. For a more general band structure the optical and electron energy surfaces will be more complicated than for the NFE case, but the principles remain.

The described procedure of confirming a calculated band structure is sometimes chosen because one cannot in general deduce the crystal momentum of an electron inside a solid from measurements of the external momentum. This is due to the fact that the component of the electron $k$-vector perpendicular to the surface $k_{\perp}$ is not conserved at the transmission through the surface. The change of $k_{\perp}$ is a function of the unknown band structure. The problem may be overcome by complementary measurements, e.g. by combining data from different crystallographic surfaces [7]. Such procedures usually give $E(k)$ in a few points only. The methods are however exact and can serve to give accurate $E(k)$ data to which a band structure can be adjusted.

A popular method to extract $E(k)$ from photoemission data to a good approximation is illustrated in Fig. 6. EDCs are recorded at normal emission for a

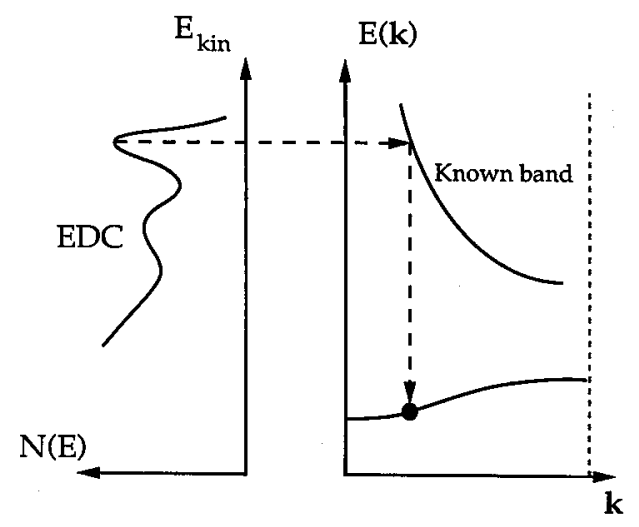

Fig. 6. The dcrivation of the band structure $E(k)$ from the measured EDC at normal emission, assuming a known final band.

series of photon energies using synchrotron radiation. The internal photoelectron energy $E_{\mathrm{f}}(k)$ is assumed to be a free electron parabola. This often turns out to be a 
good approximation over large parts of the Brillouin zone. Deviations occur, however, e.g. at zone boundaries. As seen in Fig. 6, the assumption of the known $E_{\mathrm{f}}(\boldsymbol{k})$ function allows the initial states $E_{\mathrm{i}}(k)$ to be directly determined from the recorded EDC's. The bottom of the $E_{\mathrm{f}}(k)$ parabola ("inner potential") is adjusted to reproduce maximal and minimal binding energies, corresponding to critical points in the band structure. Figure 7a shows the results by Qu et al. [8] on CdTe(110),
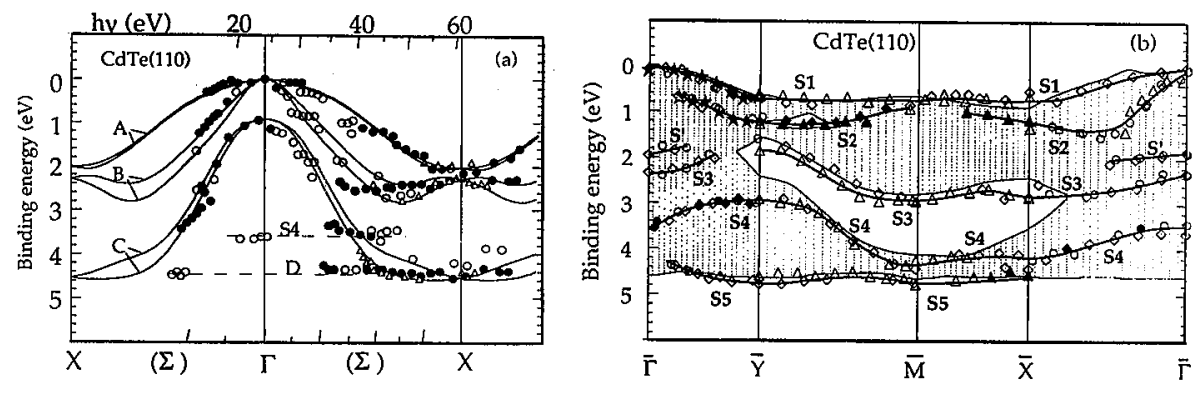

Fig. 7. (a) The band structure of CdTe derived from EDC's taken at normal emission in a range of photon energies. A semi-empirical LCAO band structure has been fitted to the experimental data. (b) Surface states and resonances deduced from the angular dependence of the EDC's. The surface projected LCAO band structure is shown as a shadowed region. The data has been taken from Ref. [8].

where the photon energy was varied in the range $20-70 \mathrm{eV}$. The so-obtained band structure is compared with an empirical tight-binding LCAO calculation.

Only part of the EDC peaks can be explained in terms of direct transitions in a bulk band structure. The binding energies of some peaks do not change when the photon energy is varied. The energies of these peaks change however as a function of the electron emission angle $\theta$, or equivalently $k_{\|}$. The excitations correspond to emission from surface states or surface resonances. These two-dimensional states have a band structure with $k$ parallel to the surface, $E\left(k_{\|}\right)$. From Eq. (13) we see that this band structure can be directly and exactly determined from EDC's recorded as a function of the detection angle $\theta$. As an example we show in Fig. 7b the results by Qu et al. [8] on the $\mathrm{CdTe}(110)$ surface. The hatched region corresponds to the bulk bands determined in Fig. 7a projected onto the surface Brillouin zone. Five surface related features S1-S5 along the edges of the surface Brillouin zone are identified. We find S3 to be situated in an absolute band gap and it is therefore termed a surface state. Other peaks, such as $\mathbf{S}$, are overlapping the bulk states and are termed surface resonances. Calculations in literature [9] on these surface related peaks show a fair agreement with experiment.

One also finds that some of the peaks in an EDC may not be associated with any of the two categories discussed above. An example of this is peak $D$ in Fig. 7a. This peak shows very little dispersion as a function of photon energy and emission angle. It is generally believed that these kinds of excitations derive from non- $k$-conserving excitations, the so-called "non-direct transitions". The initial state occurs at a critical point of high density of states, while the final state may 
be located away from the center of the final band due to lifetime effects.

Photoemission from crystals can very often be described in terms of ordinary band structure data. There are however cases when this picture breaks down. A well-known example is $\mathrm{Ni}$, for which the measured $d$-band width is considerably smaller than predicted by ground-state calculations. Moreover a strong satellite occurs below the valence band. The reason for these phenomena is that the Coulomb interactions are not small relative to the band width, which results in strong hole-hole correlation. In order to investigate the details of the band contraction Starnberg and Nilsson [10] established the band structure by first determining $E(k)$ absolutely for $11 k$-values. A semi-imperical interpolation scheme was then adjusted to these data and to the results of an ab initio band calculation. The two band structures obtained are reproduced in Fig. 8. Here there is also shown the energy difference at each $k$-point, which is attributed to the real part of a self-energy, $\operatorname{Re} \Sigma(E, k)$. Obviously there is a strong $k$-dependence in $\operatorname{Re} \Sigma(E, k)$. However, a removal of the $s p$-bands in both the theoretical as well as the experimental band structures results in a $k$-independent linear dependence $\operatorname{Re} \Sigma(E)=-0.44 E$ for the $d-d$-correlation effect. This is in agreement with theoretical studies [12].
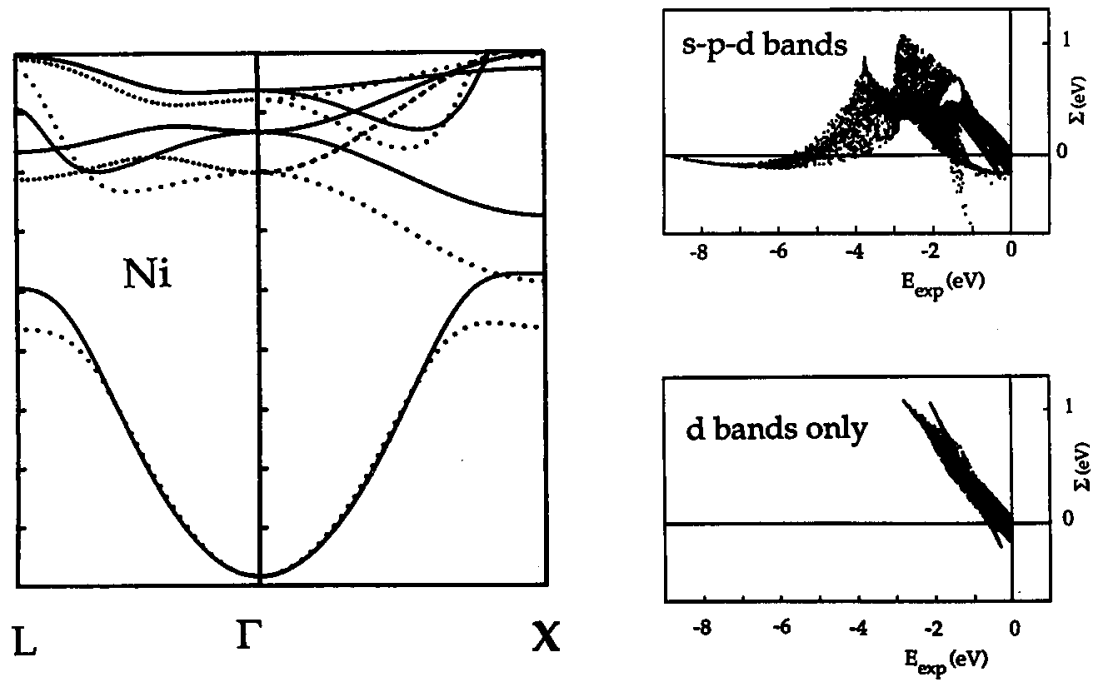

Fig. 8. The band structure of $\mathrm{Ni}$ as calculated for the ground state $(-)$, and as derived from experiment $(. .$.$) . The difference between the two band structures with and without$ $s$-p-bands is also shown (Ref. [10]).

So far we have not discussed the intensity of the EDC peaks. The intensity is given by the transition probability for direct excitation in Eq. 10. By varying the orientation of the electric field vector $e$ one finds that the intensity may be zero for a specific direction. Synchrotron radiation is advantageous here, because the radiation is highly polarised in the horizontal plane. This polarization technique may be used to determine the symmetry of bands involved in the excitation and thus 
help in the identification of the excitations. A special application is determination of the orientation of adsorbed molecules on a surface.

\subsection{Bulk versus surface contributions}

The understanding of the relative contributions to the photocurrent from the electronic states in the volume of the crystal and at the surface is of fundamental importance for the interpretation of photoelectron spectra. It is therefore worthwhile to devote a separate paragraph to this topic.

The elastic photoelectrons originate from the outermost atomic layers because the mean free path for inelastic scattering is just a few atomic layers. The question then naturally arises to what extent the observed photoelectron spectrum is influenced by the surface electronic structure. We first realise that the eigenvalues of the Schrödinger equation (Eqs. (2) and (4)) do not depend on the position coordinate $r$. Thus the eigenenergies $E_{n}(k)$ must be the same in the whole space including the surface.

For studies of surface effects the concept of local density of states is of central importance. It is defined as

$$
N(E, r)=\int \mathrm{d}^{3} k|\psi(r)|^{2} \delta\left(E-E_{n}(k)\right) .
$$

Because the wave functions $\psi(r)$ are modified in the surface region the local density of states at the surface differs from that in the bulk. The situation is schematically illustrated in Fig. 9 [13]. The total band width at the surface is the same as in

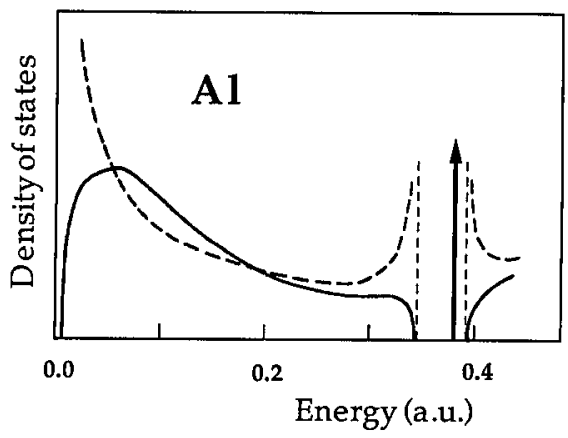

Fig. 9. The $k$-resolved density of states $(k=(0.1,0.1))$ for aluminium in the bulk $(-)$ and in the surface layer (-- ). A surface state is seen in the band gap. Data taken from Ref. [14].

the bulk. The half width of the band is however somewhat reduced at the surface due to the lower coordination number there. The loss of bulk state amplitudes in the surface region is compensated for by the appearance of new, localised states in the surface region. Narrow peaks may appear in band gaps (surface states), see Fig. 9. Broader peaks may be found in the bulk continuum (surface resonances).

The above description has important consequences for what is observed in photoemission spectra. It is explained for instance why in angle-resolved experiments the bulk band structure $E_{n}(k)$ is observed despite the fact that we are only 
testing the outermost atomic layers, where the electronic structure is strongly influenced by the surface potential. In addition surface states and resonances will be observed.

In the angle-integrated mode, and in particular for poly-crystalline samples, a density of states like spectrum is detected. Note however that the density of states will never be accurately observed because of two reasons. Firstly, the direct transitions generate a joint density of states, which is not a simple product of the initial and final density of states. Secondly, the joint density of states spectrum is modulated by transition probabilities, see Eq. (9).

\subsection{High photon energies}

The presented models so far are not directly applicable at high photon energies, say $h \nu>1 \mathrm{keV}$ (X-ray photoelectron spectroscopy, XPS). There are several factors, which make detailed band mapping of bulk and surface states not so easy in this photon region. Firstly, the angular acceptance of a conventional electron energy analyser (several degrees) causes a substantial part of the Brillouin zone to be probed. This is due to the fact that the photoelectron is excited into high order zones, which decreases the $k$-space resolution. The situation should be clear from the experimental geometry as illustrated in Fig. 10. Secondly, the fraction

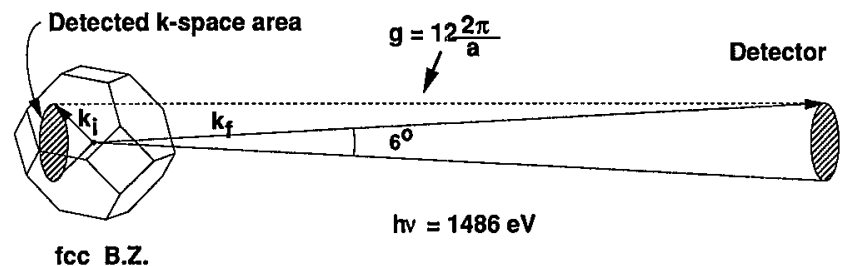

Fig. 10. The geometry of an XPS experiment. The finite angular resolution of the analyser causes an appreciable broadening in $k$-space.

of $\boldsymbol{k}$-conserving excitations decreases at high photon energies due to a decreasing Debye-Waller factor $\exp (-W)$. This is understandable if we write $W$ in the approximate form

$$
W=\frac{3 \hbar^{2}|\Delta k|^{2}}{k_{\mathrm{B}} M_{\mathrm{a}} \theta_{\mathrm{D}}^{2}} T=\frac{6 m_{\mathrm{e}}}{k_{\mathrm{B}} M_{\mathrm{a}} \theta_{\mathrm{D}}^{2}} T \hbar \omega,
$$

where $m_{\mathrm{e}}$ and $M_{\mathrm{a}}$ are the electron and atom masses, respectively, $k_{\mathrm{B}}$ - the Boltzman constant, $\theta_{\mathrm{D}}$ - the Debye temperature and $T$ - the sample temperature. The high photon energy $\hbar \omega$ can in principle be compensated for by a low temperature $T$. As the photon energy in XPS is about 10 times larger than in the VUV region we would in principle need a temperature of $T \approx 30 \mathrm{~K}$ in XPS to obtain the same results as in the VUV region. An appreciable amount of non-direct transitions, which are proportional to $(1-\exp (-W))$, will contribute to the XPS spectrum, which causes a broadening of the spectral peaks. White et al. [14] have studied the temperature dependence of valence band transitions in the $\mathrm{X}$-ray region in detail. For the VUV region the reader may consult Nilsson et al. [15]. 
The photon $k$-vector in the X-ray region cannot be completely neglected relative to the electron momentum as assumed in the VUV region. We therefore now write

$$
\boldsymbol{k}_{f}=\boldsymbol{k}_{i}+\boldsymbol{g}+\boldsymbol{q}
$$

where the photon momentum $|q|$ may cover $\approx 15 \%$ of the Brillouin zone.

Because of the $k$-averaging effect in the high photon energy region the data analysis focuses on the density of states concept. The escape length is somewhat larger in the X-ray than in the VUV-range $(\approx 20 \AA)$ and mainly the bulk states are probed. The spectrum may now be written to a good approximation as [16]

$$
\begin{aligned}
& N(E, h \nu)= \\
& C_{4} \frac{1}{\omega^{2}} \sum_{l_{\mathrm{i}}} \sum_{l_{\mathrm{f}}=l_{\mathrm{i}} \pm 1} \frac{\max \left(l_{\mathrm{i}}, l_{\mathrm{f}}\right)}{\left(2 l_{\mathrm{i}}+1\right)} N_{l_{\mathrm{i}}}\left(E_{\mathrm{i}}+h \nu\right) N_{l_{\mathrm{i}}}\left(E_{\mathrm{i}}\right)\left|M_{l_{\mathrm{i}}, l_{\mathrm{i}}}\left(E_{\mathrm{i}}, h \nu\right)\right|^{2} .
\end{aligned}
$$

Here $N_{l}(E)$ is the partial density of states of angular momentum $l$ and $M_{l_{i}, l_{\mathrm{i}}}\left(E_{\mathrm{i}}, h \nu\right)$ the radial matrix element in accelerator form, i.e the operator is $\mathrm{d} V(\boldsymbol{r}) / \mathrm{d} \boldsymbol{r}$. For a given photon energy the final density of states is approximately constant over an energy range of the order of the valence band width. We thus expect to observe the sum of the valence band partial density of states, each multiplied by a cross section. By scanning the photon energy over a large range the final state usually changes considerably. The resulting cross section variation may be used to indentify the states involved.

As an example we show in Fig. 11 the theoretical [16] and experimental [17] spectrum for the compound $\mathrm{Nb}_{3} \mathrm{Sn}$. The variation of the matrix elements over the
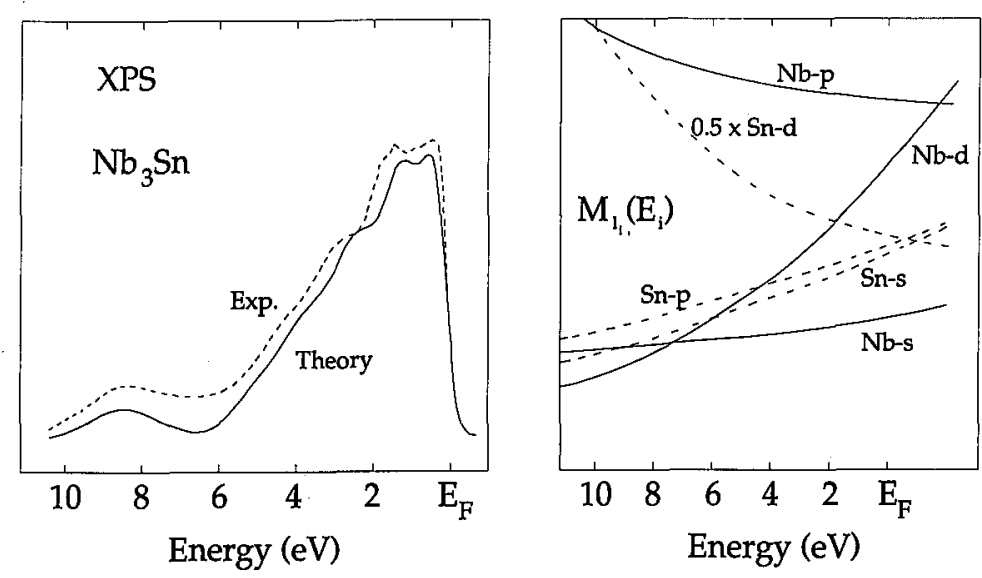

Fig. 11. Calculated [16] and measured [17] XPS spectrum of $\mathrm{Nb}_{3} \mathrm{Sb}$ using Eq. (17). The variation of the matrix elements over the valence band width is also shown.

valence band width is also shown. It is obvious that the experimental spectrum is far from proportional to the total density of states, but is well reproduced by formula (17). Further refinement can be obtained by including the modified contribution from surface layers [18]. 
The theory in this paragraph is what usually is enough to reduce experimental data. The detailed photoemission process is however far more complicated. Sometimes, and in particular in the VUV region, it may be necessary to have an insight in the full description to understand some specific results. We therefore briefly summarise the general photoemission theory in the following paragraph.

\subsection{Advanced models}

In the three-step model we associated the primary electron distribution with a first order perturbation from the light. The induced current $J$ is from a formal point of view the same as that occurring in optical absorption and electrical conduction. The internal current is in this case proportional to the electric field $E$ according to $J=\sigma E$. The equation is symbolically written and involves in principle integrations in $r$ and $q$ space. In transport theory one finds the response function $\sigma$ to be proportional to a current-current correlation function. Formally the external current is zero. To obtain an external photoelectric current we have to go to second order in the electrical field, i.e. $I=\chi E_{2}$. This is in agreement with the experimental fact that the photoelectric current is proportional to the intensity of the light. The response function is now a three-current correlation function. The golden rule in Eq. (9) may be rewritten by introduction of Green's functions [19]. In the non-interacting limit we arrive at

$$
I\left(k_{\|}, E_{\mathrm{f}}\right)=-\frac{1}{\pi} \operatorname{Im} \pi\left\langle k_{\|}\left|G_{\mathrm{f}}^{+} \Delta G_{\mathrm{i}}^{+} \Delta^{\dagger} G_{\mathrm{f}}^{-}\right| k_{\|}\right\rangle .
$$

Here $\Delta$ is the dipole operator of the light as in Eq. 10. $G_{\mathrm{f}}$ and $G_{\mathrm{i}}$ are the Green's functions for the excited electron and hole respectively. A schematic interpretation of this one-step description is given in a time-reversed picture in Fig. 12. We follow
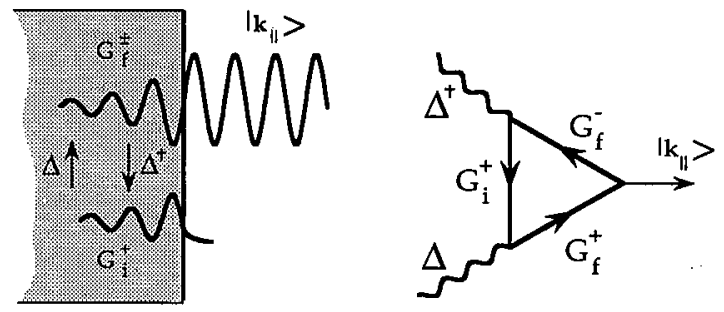

Fig. 12. The evaluation of the photocurrent using Eq. (18).

the factors from right to left in the formula (18) and associate them with the following steps. An electron in a vacuum state $\left|k_{\|}\right\rangle$enters the solid. It is multiply scattered by $G_{\mathrm{f}}^{-}$, which results in a "LEED" state $G_{\mathrm{f}}^{-}\left|k_{\|}\right\rangle$. This state is dipole deexcited by $\Delta^{\dagger}$. Holes are emitted from each atom and multiply scattered by $G_{i}^{+}$. The so-produced state is excited by $\Delta$ into the LEED state and the overlap with the vacuum state $\left|k_{\|}\right\rangle$is finally calculated. Exactly these steps may be followed in a numerical calculation. The diagram corresponding to this three-current formalism is shown in Fig. 12. One advantage with the described formulation is that lifetime 
effects conveniently can be introduced not only for the photoelectron but also for the hole state. This is done by separate renormalisations of the propagators $G_{\mathrm{i}}$ and $G_{\mathrm{f}}$. Other interactions have been discussed [20] but to our knowledge they have not yet been applied numerically.

The formalism describes a one-step process and includes surface effects. In

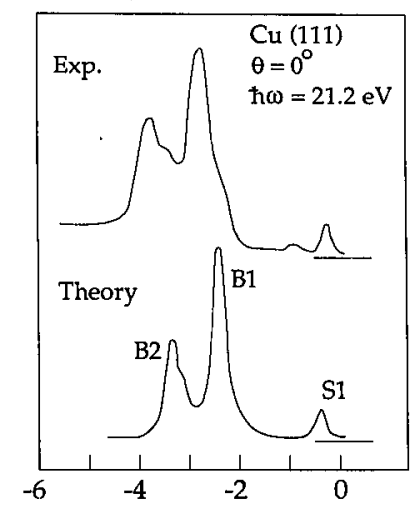

Electron energy (eV)

Fig. 13. Experimental and theoretical photoemission spectrum from $\mathrm{Cu}(111)$ taken at normal emission and with a photon energy of $21.2 \mathrm{eV}$. The comparison identifies emission from bulk states $\left(B_{1}\right.$ and $\left.B_{2}\right)$ and from a surface state $\left(S_{1}\right)$. Data taken from Ref. [20].

Fig. 13 we show experimental and theoretical results for normal emission from $\mathrm{Cu}(111)$ at $\hbar \nu=21.2 \mathrm{eV}$ [21]. The comparison identifies the two high binding energy peaks as bulk-derived. The peak just below the Fermi level is due to the surface state emission. The agreement may be improved by refining the potential by e.g. including relativistic effects and a more realistic surface potential (a step potential was used in the present calculations) [22].

\section{Photoelectron spectroscopy on core levels}

\subsection{A simple model}

As mentioned in the paragraph 2.2.2 the measured core binding energy $E_{\mathrm{B}}$ defined in Eq. (1) contains information about both the total initial as well as the total final state. It is not possible to review here all the various possible mechanisms contributing to core level spectra. Instead we will give two examples illustrating the important phenomena.

Studies of differences in binding energies are a simple application due to the different chemical bonding in the initial state. Figure 14 (lower panel) shows the $3 d$ As core spectrum for As adsorbed on an InP(100) substrate [23]. This can be considered as a monolayer of InAs on the InP surface. The spectrum can be decomposed into two constituents. These are related to two inequivalent As-atoms, 


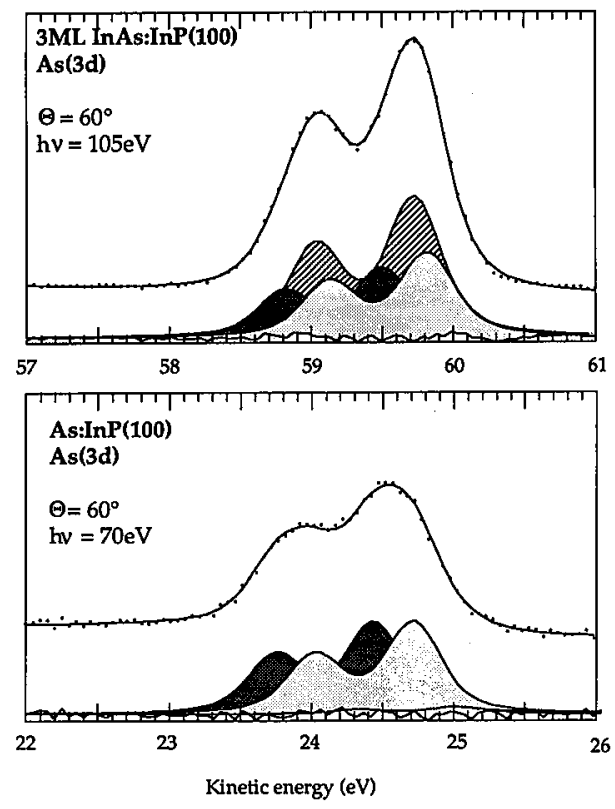

Fig. 14. The As $3 d$ core level recorded for 1 and 3 monolayers of InAs on an $\operatorname{InP}(100)$ surface. Two inequvalent surface positions and one bulk position is observed for As. From Ref. [22].

forming the so-called asymmetric dimer on the surface. The upper panel shows the same spectrum but now with 3 monolayers of $\operatorname{InAs}$ on the $\operatorname{InP}(100)$ substrate. Again the asymmetric dimer is observed but in addition we find the As signal from the underlying InAs layers. We have thus demonstrated that we can deduce geometrical information from core level studies.

\subsection{Advanced models}

An important ingredient in core level spectroscopy is the screening of the hole in the final state. Relaxation effects around the localised core hole cause the measured binding energy $E_{\mathrm{B}}$ to be several $\mathrm{eV}$ smaller than predicted by a model with "frozen orbitals". In metals, and to a large extent in semiconductors, the main relaxation effect comes from the almost complete screening of the localised positive hole by the valence electrons. The excitation spectrum does however not only reflect this "ground state" of the hole state atom, but also exhibits higher final energy states. A special effect are the low-lying hole-electron excitations around the Fermi level producing a tail on the high binding energy side of the observed peak ("infrared catastrophe"). More discrete excitations appear at higher binding energies in the form of "satellites". Finally we mention that core excitations also induce vibrational excitations, causing fine structure in the observed spectral peaks. These effects are however not always experimentally resolved in detail. 
To illustrate one aspect of the screening process we show in Fig. 15 the results obtained by $\mathrm{O}$. Björneholm et al. on $\mathrm{N}_{2}$ physisorbed on graphite [24]. The photoelectric line for ionisation of the $1 s \mathrm{~N}_{2}$ orbital gives a binding energy
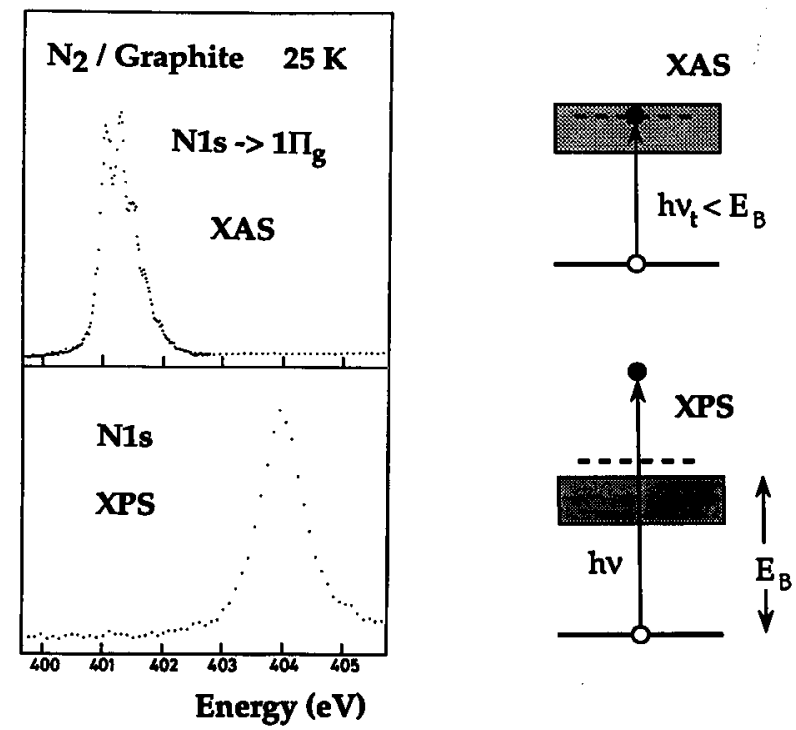

Fig. 15. X-ray photoelectron spectroscopy (XPS) and X-ray absorption spectroscopy (XAS) give different core level energies due to different screening of the core hole. From Ref. [22].

$E_{\mathrm{B}}=403.9 \mathrm{eV}$. The interaction between the adsorbate and the substrate is weak and there is no charge transfer during the excitation process from the substrate to screen the hole locally. There is howevcr some screcning due to the image charge of the hole. If the photon energy is lowered to the threshold, the $1 s$ electron is excited into an empty $1 \pi_{\mathrm{g}} \mathrm{N}_{2}$ molecular orbital. This process is observed in soft $\mathrm{X}$-ray photoabsorption (XAS), detectable in e.g. the photoyield. Figure 15 shows that the photon energy is $401.0 \mathrm{eV}$ at threshold (the fine structure is due to vibrational excitations). This energy is $2.9 \mathrm{eV}$ lower than the binding energy as measured in XPS. The difference is attributed to the fact that while the XPS hole state is unscreened, the XAS hole state is screened by the $1 \pi_{\mathrm{g}}$ electron. For chemisorbed molecules, which have a good electrical contact to the substrate, there is a finite probability for a complete screening and an XPS peak is found at the XAS threshold.

The decay processes of the excited state carry interesting information about the dynamics of the N $1 s$ core hole state [24]. The Auger spectrum from the XPS state contains contributions from both the ionic $1 \mathrm{~s}^{-1}$ state and from the neutral $1 s^{-1} 1 \pi_{\mathrm{g}}$ intermediate state. By comparing the strengths of these signals a measure of the characteristic neutralization time may be extracted. 


\section{Concluding remarks}

In this paper we have reviewed the basic ideas behind the evaluation of photoelectron spectra from solids in general. From the discussion it should be clear that today we have a good basic understanding of the photoemission process. On the other hand there are still some details in the photoemission mechanism (e.g. the temperature dependence) which need further experimental and theoretical investigations. This does not however prevent us from using photoelectron spectroscopy in a routine manner on a very broad class of materials and problems.

It has not been our intention to discuss all the variations of the method which exist today. These include e.g. photoemission using circularly polarised photons or laser light, spin-resolved photoemission, surface extended absorption fine structure (SEXAFS), spectromicroscopy, photoelectron diffraction and holography. We have discussed only a few applications, such as studies of band structures, surface states, adsorbates and overlayers. We have not touched on all the other numerous applications, such as studies of interfaces, magnetism, superconductivity, clusters, and much more.

The construction of high brilliance storage rings for synchrotron radiation now going on will provide photoelectron spectroscopy with new possibilities. For instance, it will soon become possible to record spectra in a much shorter time than today. This means that experiments not possible for the present should be feasible in the near future (e.g. coincidence experiments). On the other hand, for the spectroscopy already applied today spectra may be collected in a much shorter time, meaning that dynamical studies (e.g. chemical reactions) become feasible. The new insertion devices (undulators) will be excellent sources for providing a small focal spot for photoelectron microscopy. Development of new monochromators in the soft X-ray region and of electron spectrometers is still going on giving us an improved energy and momentum resolution as well as increased intensity. We expect to see refined photoelectron techniques applied to new problems during the 1990 's.

\section{Acknowledgements}

I am very grateful to Dr J. Kanski for critical reading of the manuscript. The Swedish Natural Science Research Council is gratefully acknowledged for financial support.

\section{References}

[1] A. Einstein, Annalen der Physik 17, 132 (1905).

[2] P. Hohenberg, W. Kohn, Phys. Rev. B 136, 864 (1964).

[3] W. Kohn, L.J. Sham, Phys. Rev. A 140, 1133 (1965).

[4] L.J. Sham, W. Kohn, Phys. Rev. 145, 561 (1966).

[5] K.W.K. Shung, B.E. Sernelius, G.D. Mahan, Phys. Rev. B 36, 4499 (1987).

[6] T. Gustafsson, L. Walldén, P.O. Nilsson, Phys. Lett. A 37, 121 (1971). 
[7] P.O. Nilsson, N. Dahlbäck, Solid State Commun. 29, 303 (1979); P. Heiman, M. Miosaga, H. Neddermeyer, Solid State Commun. 29, 463 (1979); R. Rosei, R. Lässer, N.V. Smith, R.L. Behow, Solid State Commun. 35, 979 (1980); N.E. Christensen, Solid State Commun. 38, 309 (1981); H. Neddermeyer, Solid State Commun. 40, 809 (1981).

[8] H. Qu, P.O. Nilsson, J. Kanski, L. Ilver, Phys. Rev. B 39, 5276 (1989); H. Qu, J. Kanski, P.O. Nilsson, U.O. Karlsson, Phys. Rev. B 43, 14589 (1992).

[9] M. Schmeits, Surf. Sci. 231, 389 (1990).

[10] H.I. Starnberg, P.O. Nilsson, J. Phys. F, Met. Phys. 18, L247 (1988).

[11] V.L. Moruzzi, J.F. Janak, A.R. Williams, Calculated Electronic Properties of Metals Pergamon, New York 1978.

[12] A. Liebsch, Phys. Rev. B 23, 5203 (1981); L.C. Davis, L.A. Feldkamp, Solid State Commun. 34, 141 (1980); L. Kleinman, L.A. Mednick, Phys. Rev. B 24, 6880 (1981); G. Tréglia, F. Ducastelle, D. Spanjaa.ard, J. Phys. (France) 43, 341 (1982).

[13] J.E. Inglesfield, in: Electronic Properlies of Surfaces, Ed. M. Prutton, Adam Hilger, Bristol 1984.

[14] R.C. White, C.S. Fadley, M. Sagurton, Z. Hussain, P. Roubin, D. Chandesris, J. Lecante, C. Guillot, Z. Hussain, Phys. Rev. B 35, 1147 (1987).

[15] P.O. Nilsson, L. Ilver, H.I. Starnberg, D.S.-L. Law, J. Phys., Condens. Matter 1, 6159 (1989).

[16] T. Jarlborg, P.O. Nilsson, J. Phys. C., Solid State Phys. 12, 265 (1979).

[17] H. Höchst, S. Hüfner, A. Goldmann, Solid State Commun. 19, 899 (1976).

[18] P. Steiner, S. Hüfner, Solid State Commun. 44, 619 (1982).

[19] J.B. Pendry, Surf. Sci. 57, 679 (1976).

[20] W. Bardyszewski, L. Hedin, Phys. Scri. 32, 439 (1985); C.O. Almbladh, Phys. Scr. 32, 341 (1985).

[21] P.O. Nilsson, J. Kanski, C.G. Larsson, Solid State Commun. 36, 111 (1980).

[22] R. Courths, S. Hüfner, Phys. Rep. 112, 53 (1984).

[23] J. Kanski, P.O. Nilsson, U.O. Karlsson, S.P. Svensson, Appl. Surf. Sci. 56-58, 604 (1992).

[24] O. Björneholm, A. Nilsson, A. Sandell, B. Hernnäs, N. Mårtensson, Phys. Rev. Lett. 68, 1892 (1992); O. Björmeholm, Comprehensive Summaries of Uppsala Dissertations from the Faculty of Sciences, No 367, University of Uppsala, 1992. 